\title{
An Indoor Positioning Approach using Sibling Signal Patterns in Enterprise WiFi Infrastructure
}

\author{
Xuan Du*, Kun Yang ${ }^{* \dagger}$, Xiaofeng $\mathrm{Lu}^{\dagger}$, Xiaohui Wei ${ }^{\ddagger}$ \\ ${ }^{*}$ School of Computer Science and Electronic Engineering, University of Essex, Colchester, UK \\ ${ }^{\dagger}$ ISN National Key Lab, XiDian University, Xi'an, P. R. China \\ ${ }^{\ddagger}$ School of Computer Science and Technology, Jilin University, Changchun, P. R. China \\ Email: \{xdua, kunyang\}@essex.ac.uk* ${ }^{*}$ luxf@xidian.edu.cn ${ }^{\dagger}$,weixh@jlu.edu.cn ${ }^{\ddagger}$
}

\begin{abstract}
The indoor positioning technology plays an important role in the application scenarios requiring indoor location. In this paper, the WiFi signals under modern enterprise $\mathrm{WiFi}$ infrastructure and signal patterns between coexisting access points (APs) are investigated. Sibling signal patterns are defined and processed to generate Beacon APs that have higher confidence for positioning. Then a positioning approach using Beacon APs is proposed and shows improved positioning accuracy. The proposed schemes are fully designed, implemented and evaluated in a real-world environment, revealing its effectiveness and efficiency.

Index Terms-indoor positioning, enterprise WiFi, Wi-Fi fingerprint, signal pattern, context sensing
\end{abstract}

\section{INTRODUCTION}

Indoor location is increasingly becoming one of the most important contexts for many services, such as home automation, healthcare and some other application scenarios under the scope of Internet of Things. Indoor positioning is a well-known problem and attracts a lot of research efforts in the last decade. Researchers from both academia and industry are attempting to seek the signals of opportunity for indoor positioning [1].

In recent years various positioning technologies for indoor use are emerged and range from RF (Radio Frequency) signals such as WiFi, Bluetooth, UWB (Ultra-Wide Band) and RFID (Radio-Frequency Identification) to IMU (Inertial Measurement Unit), sound, visible light and magnetic field. Meanwhile, many hybrid solutions using multiple technologies are proposed. The inertial sensors are commonly fused with other technologies to provide prediction or correction. Among these technologies, the use of WiFi signal attracts continuous attention from both academia and industries because in most cases WiFi is considered as the most ubiquitous technology. Nowadays the WiFi APs especially those of enterprise WiFi infrastructure have been deployed in most public places and almost everyone has a WiFi-compatible smartphone in their pocket. The existing WiFi APs can be used as positioning beacons and smartphones are targeted mobile handheld, so dedicated hardware is not required for positioning purpose and smartphone naturally works as the interface for users to interact with location-based services [2] [3] [4].

Fingerprinting is a widely-used technique for positioning using WiFi. The WiFi fingerprint is defined as a mapping of APs' received signal strength (RSS) observations to locations. The RSS observation is a vector of RSS from different APs that are reachable at the location. Fingerprint-based positioning usually consists of two stages: offline site survey (training) then online positioning. Site survey is conducted by a WiFicompatible mobile handheld (MH) to collect WiFi fingerprint at every reference point (RP) in the interesting area. The fingerprints collected at all the reference points constitute a fingerprint database to serve online positioning. In positioning phase, the real-time RSS observed by a WiFi-compatible MH is compared with RSS observations in fingerprint database to find the best-matched one. The corresponding location of the best-matched RSS observation is returned as user's location [5] [6] [7].

Signal fluctuation and heterogeneous mobile devices lead to inconsistent signal measurements and eventually location errors. To mitigate the inconsistency, the signals are observed from a broader view, such as temporal and spatial observation. Kim et al. [8] propose a smartphone-based pedestrian-tracking system using WiFi. In their system, an approach named Peakbased Wi-Fi Fingerprinting (PWF) is proposed to overcome RSS variance problem. The PWF method improves the system accuracy by detecting the signal strength peak from temporal signal patterns but has the problem of potential missing scan of peak values. In Walkie-Markie [9] the WiFi-defined landmark (WiFi-Mark) is defined as a location where the trend of an AP's RSS reverses, i.e., as the user moves along a pathway, the RSS reading is changing from increasing to decreasing. This approach by examining the RSS trend instead of RSS readings turns out that no matter how the devices are different and how the user is holding the device, the WiFi-Mark occurs at the same location.

In this paper, the signals are observed from the view of coexisting visible APs and sibling signal patterns are defined. Based on sibling signal patterns the Beacon APs with higher confidence are generated. An indoor positioning approach using Beacon APs is proposed. In summary, the major contributions of this paper are as follows.

- Analyze the WiFi signals under modern enterprise WiFi infrastructure and define sibling signal patterns.

- Sibling signal patterns are processed to generate Beacon APs and RSS of Beacon APs are mapped to the indoor map.

- Propose a positioning approach using Beacon APs to reduce the impact of signal fluctuation and eventually 
improve the positioning accuracy.

This paper is organized as follows. The analysis of signals and concept of sibling signal pattern are introduced in Section II. Section III presents the sibling signal patterns processing schemes to generate Beacon APs. The positioning approach using Beacon APs is presented in Section IV. Performance evaluation and analysis of the proposed positioning approach are discussed in Section $\mathrm{V}$ before the paper concludes in Section VI.

\section{Analysis Of Signal Patterns}

To decide how to use the WiFi signals properly and efficiently, analyzing WiFi signals is conducted in our experimental site, i.e., main campus building at University of Essex Colchester Campus. Each single AP is uniquely identified by the Basic Service Set Identifier (BSSID). Around 200 APs are observed during the 25 seconds movement. The Fig. 1 (left) pie chart shows the percentage of observed APs with different appearance frequency (denoted by N) when a mobile handheld is moving along a corridor for 25 seconds. With the increase of appearance frequency, the number of APs decreases significantly. Around one-third of APs are observed only once and about a quarter of APs are observed for more than 3 times. The AP appearing fewer times normally means its signal strength are weak and it can be observed only within a short period. The Fig. 1 (right) bar chart shows the distribution of observed APs in $2.4 \mathrm{GHz}$ and $5 \mathrm{GHz}$ frequency band respectively. APs of $5 \mathrm{GHz}$ dominates the observations with smallest and largest appearance frequency, which reveals that $5 \mathrm{GHz}$ channels may be less crowded and weak signals in $5 \mathrm{GHz}$ are more likely to be observed than that in $2.4 \mathrm{GHz}$ [10].
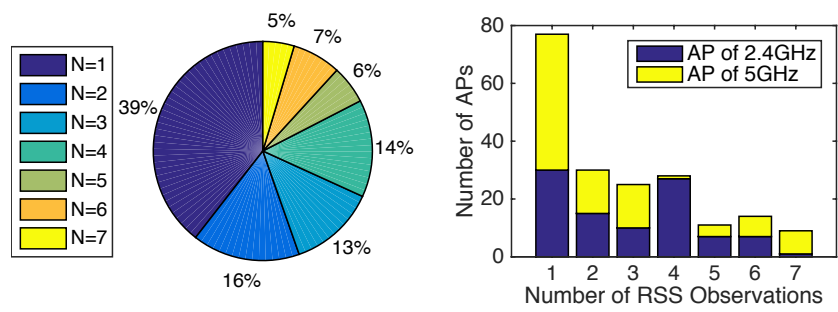

Fig. 1. Occupancy of 195 observed APs with a different number of observation (left) and frequency band (right).

Through empirical investigation of the WiFi networks in our experimental site, one major reason caused the excessive number of APs is the virtual access point (VAP) functionality of enterprise WLAN infrastructure, which allows one physical AP to have multiple separate WLAN with its own BSSID and SSID (service set identifier). Another reason is the APs Simultaneous Dual-Band functionality that enables each WLAN to operate in both $2.4 \mathrm{GHz}$ and $5 \mathrm{GHz}$ frequency band, which uses two different BSSID but the same SSID. In this paper, we name the APs from the same physical AP as sibling APs.

The idea of fingerprint-based positioning was originally proposed based on the principle that several APs located at different places can provide distinguishing signal strength observations in different observation point. However nowadays the APs observed by users are heterogeneous and some of them are coming from the same physical AP at the same location. Thus, the concept of sibling signal patterns (SSP) is proposed to describe the correlation between the sibling APs.

The sibling signal patterns are investigated in our work. Since the VAPs from the same physical AP share the same radio frequency, which has been verified in our experiment, we believe the signals of these VAPs suffer similar interference in the environment and the RSS of them at the same place are supposed to be the same. This thought is proved by the real data collected in our experiment, which is depicted in the Fig. 2. The top and bottom bar chart in the Fig. 2 respectively shows the time-series RSS observations of VAPs operating in both $2.4 \mathrm{GHz}$ and $5 \mathrm{GHz}$ frequency band, which are observed by a mobile device moving along a corridor for 25 seconds. In total, there are 9 BSSID coming from the same physical AP. The bar charts clearly illustrate that the RSS of VAPs coming from the same physical AP and operating in the same frequency band are almost identical at the same time and location. Apart from fluctuation caused by noisy environment, the minor difference of RSS between VAPs may also come from the measurement tolerance in the mobile device. At certain locations, the APs with weak signals are not observed. In general, the observations in $5 \mathrm{GHz}$ has better integrity than that in $2.4 \mathrm{GHz}$.
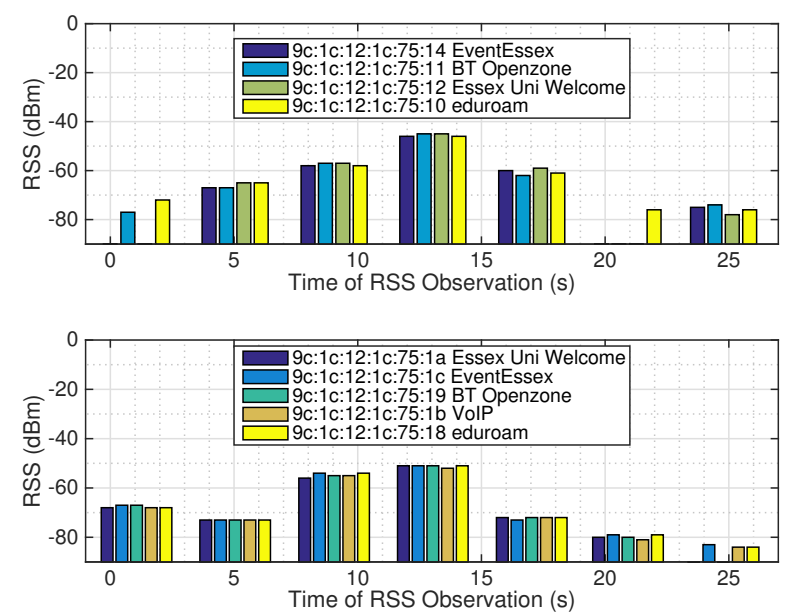

Fig. 2. The RSS observations of VAPs operating in $2.4 \mathrm{GHz}$ (top) and $5 \mathrm{GHz}$ (bottom) frequency band from the same physical AP when moving along a corridor for 25 seconds.

\section{Sibling Signal Pattern Processing}

A path segment is a directed line segment consisting of a sequence of points in the corridor and defined by a start node and an end node, as illustrated in Fig. 3. When the mobile handheld of the surveyor is moving along a path segment, a set of continuously observed raw RSS samples from surrounding APs are collected. The RSS sample set collected at path segment $e_{i}$ from $K$ number of APs is 
TABLE I

PART OF THE NOTATIONS

\begin{tabular}{ll}
\hline Notation & Description \\
\hline$e$ & A path segment \\
$\mathbf{S}_{i}$ & RSS values observed from $e_{i}$ \\
$\mathbf{T}_{i}$ & Observation timestamps from $e_{i}$ \\
$K$ & Number of APs \\
$N$ & Number of observations \\
$\mathbf{x}_{k}$ & RSS values from $k^{t h} \mathrm{AP}$ \\
$x_{n}$ & RSS value of the $n^{t h}$ observation \\
$b_{k}$ & BSSID of $k^{t h} \mathrm{AP}$ \\
$f_{k}$ & Frequency of $k^{t h} \mathrm{AP}$ \\
\hline
\end{tabular}

denoted as $\mathbf{S}_{i} \equiv\left(\mathbf{x}_{1}, \ldots, \mathbf{x}_{K}\right)$, where $\mathbf{x}$ denotes a vector with $N$ observations of time-series RSS values $x$, denoted as $\mathbf{x} \equiv\left(x_{1}, \ldots, x_{N}\right)^{\mathbf{T}}$. The number of observations $N$ can be different in $\mathbf{x}_{k}$ from different APs. $\mathbf{S}_{i}$ are collected together with the corresponding observation timestamp of the RSS values, denoted as $\mathbf{t} \equiv\left(t_{1}, \ldots, t_{N}\right)^{\mathbf{T}}$ and $\mathbf{T}_{i} \equiv\left(\mathbf{t}_{1}, \ldots, \mathbf{t}_{K}\right)$. Meanwhile, the BSSID and frequency of APs are also recorded and denoted as $\mathbf{b} \equiv\left(b_{1}, \ldots, b_{K}\right)^{\mathbf{T}}$ and $\mathbf{f} \equiv\left(f_{1}, \ldots, f_{K}\right)^{\mathbf{T}}$.

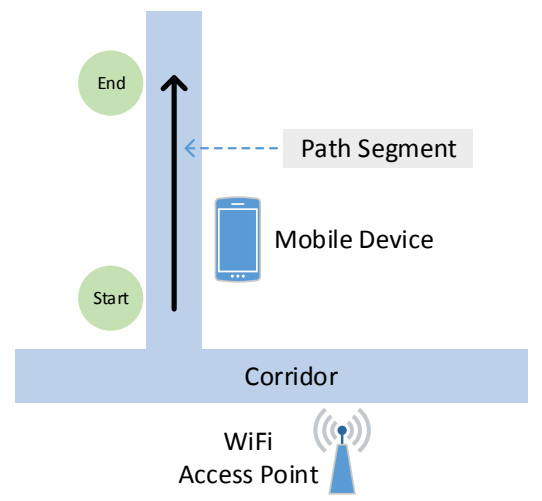

Fig. 3. Illustration of path segment.

Process Motivation. The analysis of WiFi signals concludes that the overall quantity of available APs is massive and the appearance frequency of APs differs greatly. Using all the observed APs for positioning leads to high computation complexity and may cause extra errors. Thus, the overall AP quantity need to be reduced and quality of AP needs to be evaluated. Eventually, only a small portion of high-quality WiFi signals can be used for positioning.

The analysis reveals that the VAPs from the same physical AP present similar observed RSS. Thus, the concept of Beacon AP is proposed and a Beacon AP is defined as a delegation to the VAPs from the same physical AP and in the same frequency band. The fingerprints of Beacon APs are finally saved and used for positioning. The steps of processing fingerprints are as the pseudocode in Algorithm 1.

The Sibling Signal Pattern Processing Algorithm takes the raw observations from each path segment as input and generates Beacon APs of the path segment as output. Firstly, considering strong signal shows higher confidence than weaker

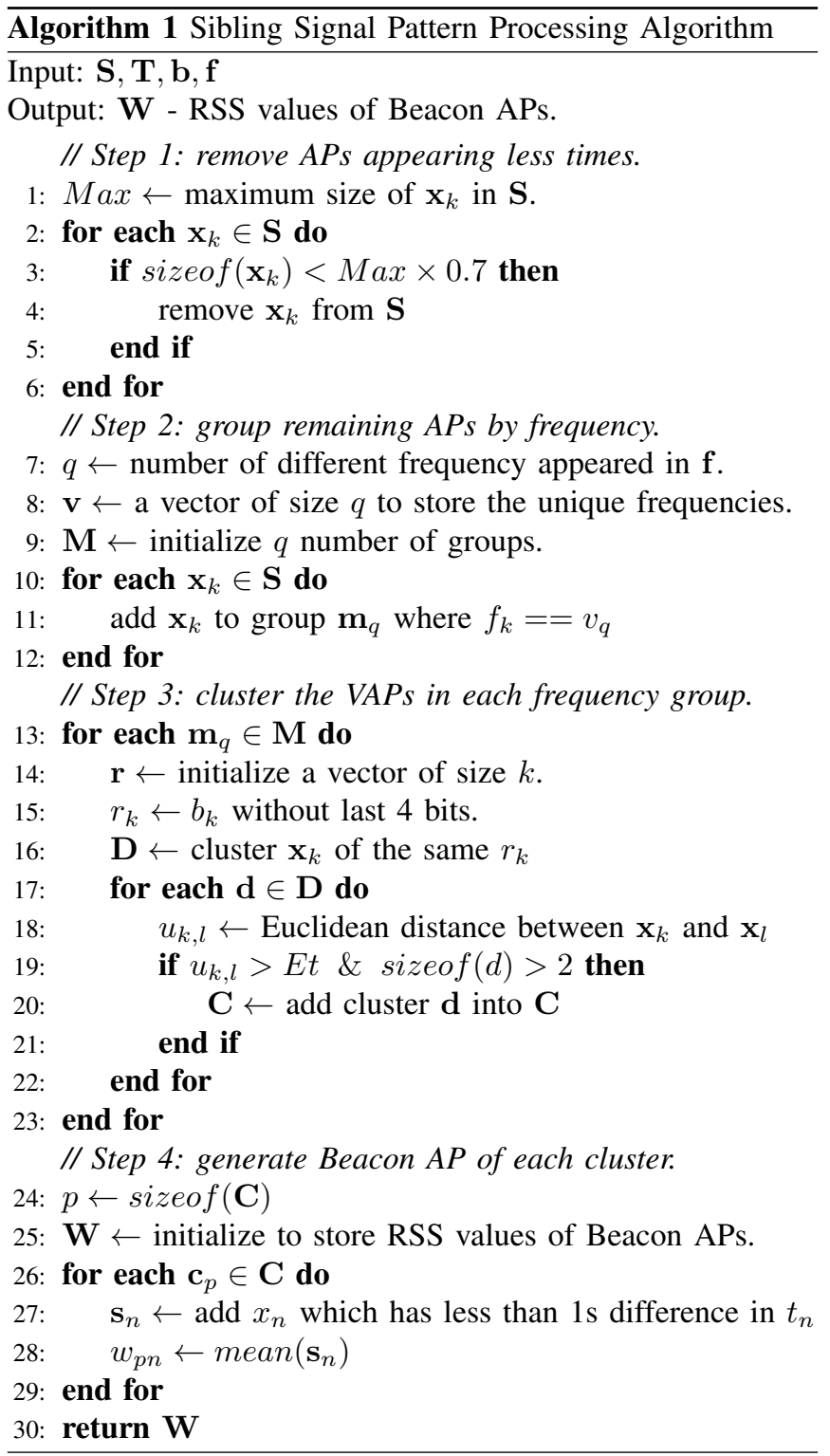

one and APs appearing fewer times are relatively weak, therefore APs with low appearance frequency are filtered out. A threshold indicating appearance frequency is used to control the APs need to be removed. Secondly, the remaining APs are divided into groups by their frequency. Each group may contain APs of the same frequency but from more than one physical APs because more than one APs may operate at the same frequency. Thus, thirdly, the APs in each group are grouped again to form the cluster of APs which are at the same frequency and from the same physical AP, i.e., the VAPs from a physical AP. The approach to cluster the VAPs is based on empirical practice and theoretical verification. Finally, each VAP cluster is processed to generate a Beacon AP. The processed signals in different stages are illustrated in Fig. 4 using one path segment of our experimental site as an example.

Clustering VAPs. The empirical practice is that the BSSID 
of VAPs from the same physical AP normally has certain correlation. The MAC address of AP is usually used as the BSSID and contains 48 bits. The MAC addresses of VAPs in the same physical AP are usually the same except last 4 bits. Thus, VAP key is defined as \{BSSID[1:44], Frequency\}, for example, \{24:de:c6:c3:5b:b, 2412\}. The VAPs are identified and clustered based on their keys. To make sure the empirical practice always work correctly, the clustered VAPs are verified by checking the similarity between signal patterns. The similarity between signal temporal patterns from two APs is determined based on the Euclidean distance between them, which is denoted as

$$
u_{k l}=\sqrt{\sum_{m=1}^{M}\left\{\mathbf{x}_{k, m}-\mathbf{x}_{l, m}\right\}^{2}}
$$

where $\mathrm{M}$ is the number of RSS observations in which both APs appear at the same timestamp and in the same channel. If the Euclidean distance between any two APs in the cluster is larger than the threshold, the VAPs in this cluster may not be from the same physical AP and this cluster is not used for later work.

Beacon AP Generation. Before the clustered VAPs are used to generate Beacon AP, the number of VAPs in each cluster is checked and if it is too few we can believe the VAPs in this cluster have less opportunities to be spotted. Our experiment also shows the VAPs in the cluster of small size have relatively weak signal strength. Thus, only the clusters with more than two VAPs are kept to generate Beacon APs. Finally, in each cluster, if the observations of different VAPs are captured in the same time, the mean of their RSS values is used as the RSS value of Beacon AP at that timestamp, which is the final data used for positioning.
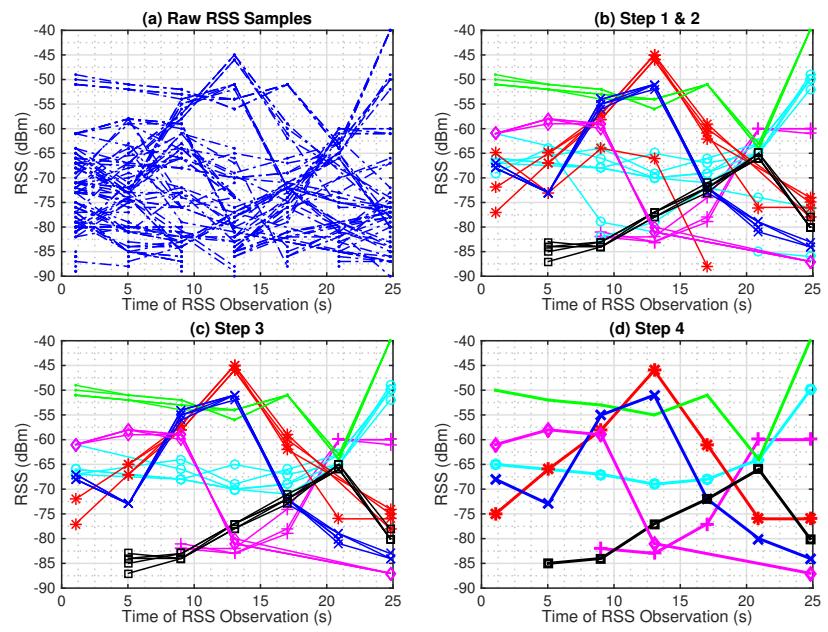

Fig. 4. Illustration of processing signal patterns from raw RSS samples observed from APs in one path segment of our experimental site.

\section{Positioning USING BeACON APS}

The system consists of three modules: site survey, SSP processing and positioning, as the system architecture depicted in Fig. 5. In the offline phase, a dedicated surveyor holding a $\mathrm{MH}$ running site survey module is walking along the corridors to collect raw observations. Then the server-side SSP Processing module processes the collected raw data to produce Beacon AP RSS map. In the online phase, positioning module is running on the targeted $\mathrm{MH}$ to observe the real-time signal and compare it with the Beacon AP RSS map in the database to compute the location of the $\mathrm{MH}$.

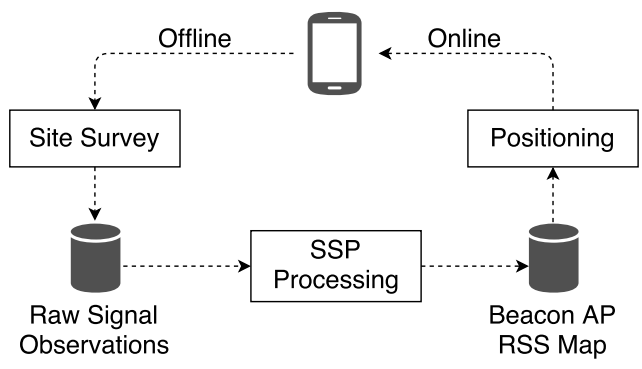

Fig. 5. System Architecture.

The RSS of Beacon APs processed from raw signal observations are mapped to the indoor map to form RSS map of Beacon APs. To map the RSS of Beacon APs to the spatial locations, the reference points (RP) are employed like most fingerprint-based approaches [11]. The RSS map consists of RSS from Beacon APs at reference points of each path segments. The RSS from $P$ number of Beacon APs at $N$ number of reference points of path segment $e_{i}$ is denoted as

$$
W_{i}=\left(\begin{array}{ccc}
w_{1,1} & \cdots & w_{1, N} \\
\vdots & \ddots & \vdots \\
w_{P, 1} & \cdots & w_{P, N}
\end{array}\right)
$$

where $w_{p n}=-90$ if Beacon $\mathrm{AP} p$ is not observed at $\mathrm{RP} n$, because the weakest signal observed is close to -90 but no less than -90. The number of RPs of each path segment is determined based on its length. Since Beacon APs are elected based on path segment and just represent signal patterns over that path segment, size and identity of Beacon APs in different path segments are not consistent.

A positioning algorithm by matching the RSS observations of Beacon APs using K-Nearest Neighbors (KNN) algorithm is used to find the best-matched RP, as the pseudocode in Algorithm 2. The RSS Matching Algorithm takes current RSS observation and RSS map as inputs and outputs the estimated location. Each RP in the RSS map is retrieved and the mutual APs appeared in both the RP and current observation are obtained. The number of mutual APs is checked against the total number of APs at this RP, because the RP is possible to be the estimated location only when most of the APs in the $\mathrm{RP}$ are present. A threshold is used to control the proportion of mutual APs. If the RP passes the AP proportion check it becomes a candidate RP and the Euclidean distance between the RSS vectors from mutual APs is calculated. The Euclidean distance divided by the number of APs is used as the similarity of this RP. Finally, the candidate RP of smallest Euclidean distance is selected as the estimated location. 


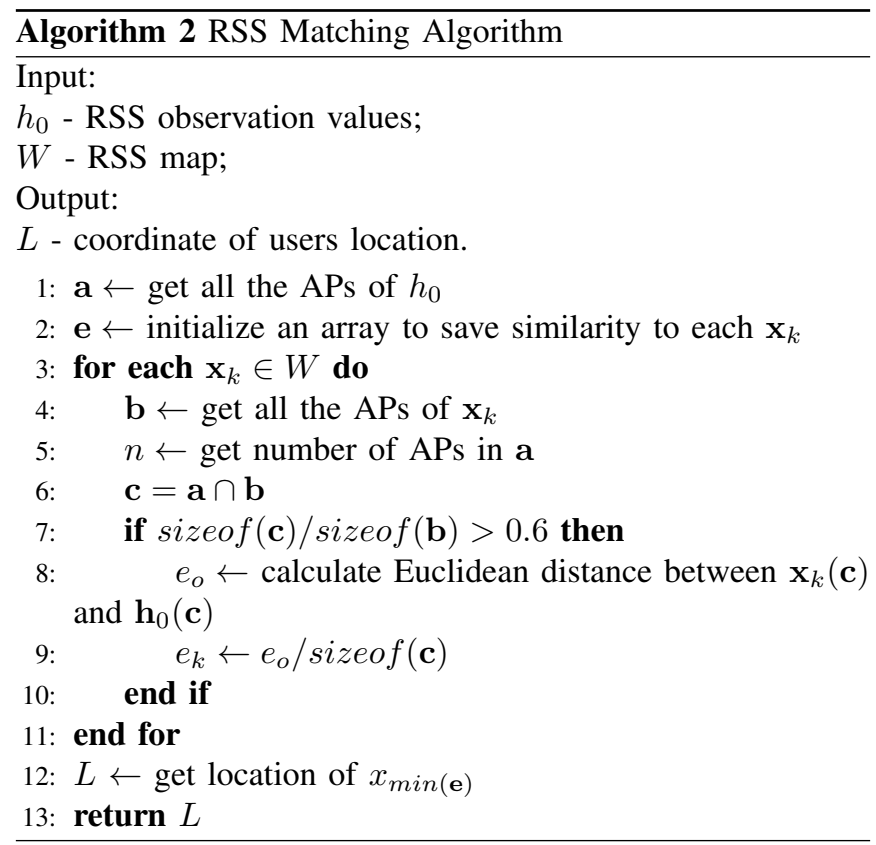

\section{Evaluation}

\section{A. Evaluation Methodology}

We develop the prototype in which the site survey and positioning modules are implemented on Android platform and the SSP processing module on Matlab. All the data collected on Android smartphone are saved into SQLite database, which is retrieved by Matlab to process and then send back to the smartphone to eventually fulfill positioning. The experiments are conducted on the 5 th floor of the central campus building at the University of Essex Colchester Campus, which is about 50 meters length, as depicted in Fig. 6 (left). Since the positioning performance of movement in corridors is our main concern and corridors are just all accessible area, the office or seminar rooms are not covered in our experiment. The site is covered by about 50 wireless APs (250 VAPs) of Aruba which belongs to the campus WiFi infrastructure mounted on the ceiling.

The system is evaluated from several aspects and the major benchmark is the raw RSS scheme, i.e., the traditional approach used by many systems. Two positioning schemes, raw RSS and Beacon AP RSS, are implemented in the Android app and allow users to switchover in the setting menu. In raw RSS scheme, reference points are sampled approximately every 35 meters and each RP is trained for around 10 seconds. In the Beacon AP RSS scheme involving sibling signal patterns, the space is profiled by site survey at a steady speed (about 1 meter per second), as shown in Fig. 6 (middle). In positioning stage, no matter what scheme is applied, the positioning result is shown as a red dot on the map, as shown in Fig. 6 (right). To evaluate the accuracy, ground truth is marked by tapping the real location on the map and saved into positioning logs. Location error is defined as the Euclidean distance from the estimated location to ground truth. Meanwhile, some other data such as real-time observed APs and candidate RPs in positioning algorithm are also recorded into positioning log sat in SQLite database.
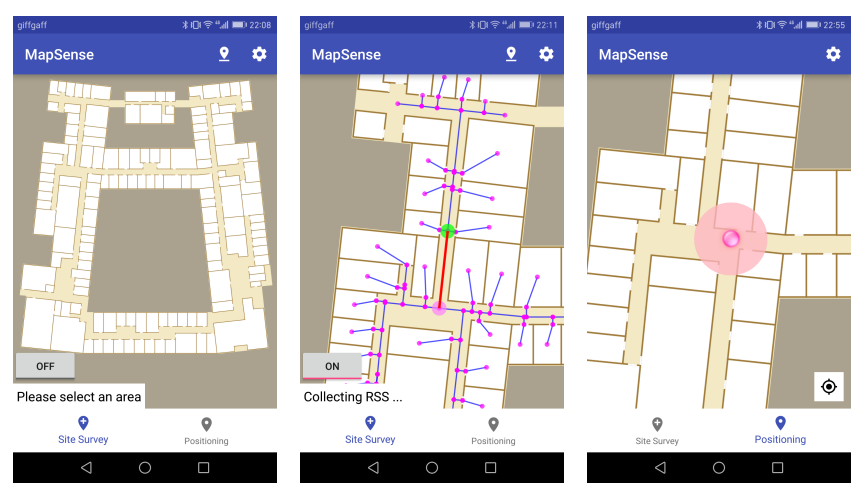

Fig. 6. Illustration of indoor map of the experimental site (left), the user interface of site survey (middle) and positioning result (right) shown on Android app.

\section{B. Analysis of Positioning Accuracy}

As the RSS variance is a major problem of WiFi-based positioning, the effectiveness of sibling signal pattern is evaluated against different device setups. As descripted in Table II, two devices with different physical setup and software setting are employed to evaluate positioning performance while the site survey is conducted by Huawei P9. As shown in Fig. 7, in this test Beacon AP RSS leads raw RSS in the overall performance. Beacon AP RSS scheme provides positioning result within 2 meters from ground truth in over $90 \%$. The maximum location error distance is reduced by 2 meters to just over 3 meters against raw RSS scheme. The key point is how two different schemes are effected in M4 setup. Based on raw RSS the location error is amplified when MH running positioning module is not the $\mathrm{MH}$ performed site survey. Under such situation using Beacon AP RSS, the positioning accuracy is also effected but just a small drop, which reveals Beacon APs generated by sibling signal patterns are more robust to device variance.

TABLE II

DEVICE SETUPS

\begin{tabular}{|c|c|c|c|}
\hline Setup & Device Model & Platform Version & WiFi Scan Frequency \\
\hline P9 & Huawei P9 & Android 6.0 & every 4s \\
\hline M4 & Xiaomi 4 & Android 5.0 & every 6s \\
\hline
\end{tabular}

Since the Beacon APs are generated based on RSS observations collected in movement while raw RSS is collected in stationary, the positioning accuracy is evaluated in both stationary and moving state. As illustrated in Fig. 8, Beacon AP RSS scheme offers the best performance when the $\mathrm{MH}$ is in movement. When it is used in stationary the performance decreases a bit and its maximum error distance overtakes raw RSS scheme in stationary. We believe the Beacon AP RSS performs better in movement because the Beacon APs and their RSS are generated from RSS observations in movement. While raw RSS in movement performs worst. From which 


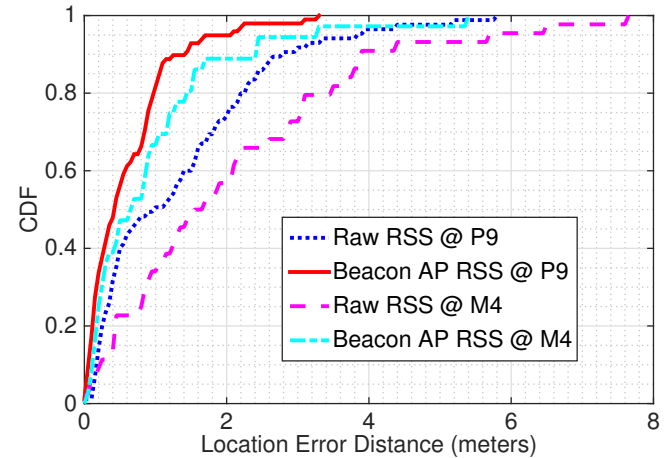

Fig. 7. CDF of different device setups.

we can see Beacon AP RSS scheme are more suitable for application scenarios of moving $\mathrm{MH}$.

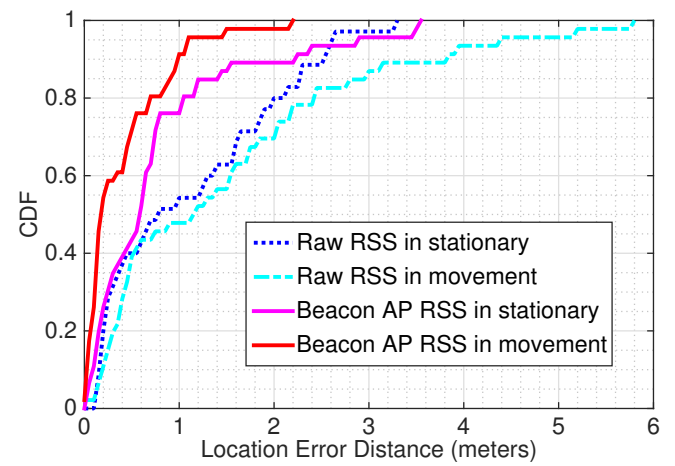

Fig. 8. CDF of different usage scenarios.

\section{Efficiency Comparison}

Apart from positioning accuracy, the system efficiency is becoming another important concern in the real-world deployment. In the site survey stage, the Beacon AP RSS approach can cover corridor of 20-meter length for less than 30 seconds. However, the traditional predefined RP iteration method can take more than 5 minutes depending on the grid size of RPs and sample size at each RP. In the positioning stage, the RSS matching algorithm spends most computational resource and energy. The computation cost of RSS matching algorithm is mainly depending on the number of candidate RPs to search for the best-matched location. As shown in Fig. 9, the Beacon AP approach takes less than half of candidate RPs of raw RSS, which can reduce the computation cost accordingly.

\section{CONCLUSION ANd Future Work}

In this paper, the correlation of signals between sibling APs is analyzed. A positioning approach using Beacon APs generated based on sibling signal patterns is proposed and shows better performance compared with a traditional approach. In the experiments, for the path segment which is relatively shortdistance, it has just a few number of RSS observations, in which case the Beacon AP cannot be generated, so other

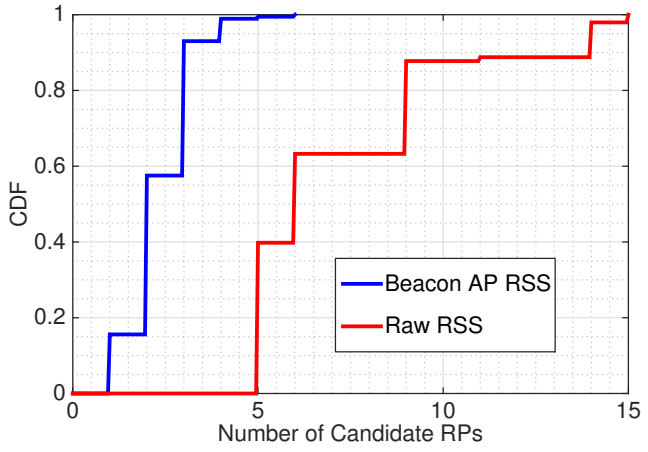

Fig. 9. CDF of the number of candidate RPs from positioning log.

strategies like limiting the minimum length of path segment are necessary to be considered in the future work.

\section{ACKNOWLEDGMENT}

The work presented in the paper was partly funded by UK EPSRC Project DANCER (EP/K002643/1), EU FP7 Projects CROWN (GA-2013-610524) and Natural Science Foundation of China (Grant No. 61572389 / 61620106011).

\section{REFERENCES}

[1] C. Perera, A. Zaslavsky, P. Christen, and D. Georgakopoulos, "Context aware computing for the internet of things: A survey," IEEE Communications Surveys Tutorials, vol. 16, no. 1, pp. 414-454, First 2014.

[2] S. He and S.-H. Chan, "Wi-fi fingerprint-based indoor positioning: Recent advances and comparisons," Communications Surveys Tutorials, IEEE, vol. PP, no. 99, pp. 1-1, 2015.

[3] D. Lymberopoulos, J. Liu, X. Yang, R. R. Choudhury, S. Sen, and V. Handziski, "Microsoft indoor localization competition: Experiences and lessons learned," GetMobile: Mobile Comp. and Comm., vol. 18, no. 4, pp. 24-31, Jan. 2015.

[4] S. He, S. H. G. Chan, L. Yu, and N. Liu, "Fusing noisy fingerprints with distance bounds for indoor localization," in 2015 IEEE Conference on Computer Communications (INFOCOM), April 2015, pp. 2506-2514.

[5] P. Bahl and V. Padmanabhan, "Radar: an in-building rf-based user location and tracking system," in INFOCOM 2000. Nineteenth Annual Joint Conference of the IEEE Computer and Communications Societies. Proceedings. IEEE, vol. 2, 2000, pp. 775-784 vol.2.

[6] A. Au, C. Feng, S. Valaee, S. Reyes, S. Sorour, S. Markowitz, D. Gold, K. Gordon, and M. Eizenman, "Indoor tracking and navigation using received signal strength and compressive sensing on a mobile device," Mobile Computing, IEEE Transactions on, vol. 12, no. 10, pp. 2050 2062, Oct 2013.

[7] C. Feng, W. S. A. Au, S. Valaee, and Z. Tan, "Received-signal-strengthbased indoor positioning using compressive sensing," IEEE Transactions on Mobile Computing, vol. 11, no. 12, pp. 1983-1993, Dec 2012.

[8] Y. Kim, H. Shin, Y. Chon, and H. Cha, "Smartphone-based wi-fi tracking system exploiting the rss peak to overcome the rss variance problem," Pervasive Mob. Comput., vol. 9, no. 3, pp. 406-420, Jun. 2013.

[9] G. Shen, Z. Chen, P. Zhang, T. Moscibroda, and Y. Zhang, "Walkiemarkie: Indoor pathway mapping made easy," in Proceedings of the 10th USENIX Conference on Networked Systems Design and Implementation, ser. nsdi'13. Berkeley, CA, USA: USENIX Association, 2013, pp. 8598.

[10] J. Shi, L. Meng, A. Striegel, C. Qiao, D. Koutsonikolas, and G. Challen, "A walk on the client side: Monitoring enterprise wifi networks using smartphone channel scans," in IEEE INFOCOM 2016 - The 35th Annual IEEE International Conference on Computer Communications, April 2016, pp. 1-9.

[11] C. Feng, W. Au, S. Valaee, and Z. Tan, "Compressive sensing based positioning using rss of wlan access points," in INFOCOM, 2010 Proceedings IEEE, March 2010, pp. 1-9. 